\title{
EMIL: The Energy Materials In Situ Laboratory Berlin
}

\author{
K. Lips ${ }^{1}$, D.E. Starr ${ }^{1}$, M. Bär ${ }^{1,2}$, T. F. Schulze ${ }^{1}$, F. Fenske ${ }^{1}$, S. Christiansen ${ }^{1}$, R. van de Krol$^{1}$, S. Raoux ${ }^{1}$, G. \\ Reichardt $^{1}$, F. Schäfers ${ }^{1}$, S. Hendel ${ }^{1}$, R. Follath ${ }^{1,3}$, J. Bahrdt ${ }^{1}$, M. Scheer ${ }^{1}$, G. Wüstefeld ${ }^{1}$, P. Kuske ${ }^{1}$, M. \\ Hävecker $^{1}$, A. Knop-Gericke ${ }^{4}$, R. Schlögl ${ }^{4}$, B. Rech ${ }^{1}$ \\ ${ }^{1}$ Helmholtz-Zentrum Berlin für Materalien und Energie, Berlin, Germany \\ ${ }^{2}$ Inst. f. Physik und Chemie, Brandenburgische TU Cottbus-Senftenberg, Cottbus, Germany \\ ${ }^{3}$ Paul-Scherrer Institut, Villingen, Switzerland \\ ${ }^{4}$ Inorganic Chemistry Department, Fritz-Haber-Institute of the Max-Planck-Society, Berlin, Germany
}

\begin{abstract}
In a concerted effort, the Helmholtz-Zentrum Berlin für Materialien und Energie GmbH (HZB) and the Max Planck Society (MPG) will develop, install, and operate EMIL, the Energy Materials In situ Laboratory, which will be a unique facility at the BESSY II synchrotron light source in Berlin, Germany. EMIL will be dedicated to the in situ and in operando $\mathrm{X}$-ray analysis of materials and devices for photovoltaic applications and of (photo)catalytic/photo-electrochemical processes. EMIL provides access to soft and hard $\mathrm{X}$-rays in an energy range of $80 \mathrm{eV}-10 \mathrm{keV}$, and comprises all characterization and deposition facilities in one integrated ultrahigh vacuum system. EMIL allows studying solar energy converting devices including photovoltaic (PV) structures based on silicon- and compound semiconductors, hybrid organic/inorganic heterojunctions, emerging organo-metal halide perovskites as well earth-abundant materials for solar fuel production (water splitting).
\end{abstract}

\section{INTRODUCTION}

One of the main challenges for today's global society is a reliable, cost-effective and environmentally-friendly supply of energy. Renewable energies will likely carry the major load within a future sustainable energy system and photovoltaics (PV) as well as photo(electro)chemical fuel production will play important roles. Technology development and mass production have pulled down the costs of PV during the past decades. However, in order to accommodate the necessary economic constraints to massively implement PV on a global scale, substantial cost reductions are further needed and the integration of PV in a supply system tackling the fluctuating availability of solar radiation is a must. This additionally calls for economically suitable solutions for energy storage/fuel production.

To achieve these ambitious goals, a knowledge-based approach to materials research will become necessary with a fast and direct feedback between sophisticated analytics and state-of-the-art deposition systems which are capable to process complete devices. In particular, it requires the deliberate design of materials and interfaces. Thus, the understanding of the electronic, optical, chemical, and structural properties of materials involved becomes very important. The relevant active interfaces that determine device operation are often not directly accessible for spectroscopy since they are buried under a contact material or a thin liquid film (as e.g. in a solar cell heterojunction or water on top of a photocatalytically active surface). Such buried interfaces usually become accessible by removing the respective layers. However, through this procedure, changes to the interface properties will be induced leading to strong deviations with respect to detected interface properties (e.g. induced by missing surface dipoles or due to contamination).

An approach to tackle this problem is the coupling of synchrotron-based X-ray characterization techniques with a variety of information depths - such as X-ray photoelectron spectroscopy and -microscopy (XPS/XPEEM), as well as Xray absorption (XAS) and emission/fluorescence (XES/XRF) spectroscopy, with relevant deposition and post-treatment capabilities in one dedicated vacuum system.

Despite the detailed information that can be obtained through synchrotron-based spectroscopy, two main limitations are currently restricting the broad application of synchrotron light sources in energy materials research worldwide: (i) The feedback times between spectroscopy at the synchrotron and performance results for materials and device development are too long to allow for a truly knowledge-based design due to limited access to synchrotron sources. (ii) The limited X-ray energy range at most beamlines does not allow a continuous use of soft and tender X-rays as would be mandatory to study surfaces and buried interfaces on the same sample without moving to another experimental setup.

\section{EMIL'S APPROACH}

The aforementioned scientific challenges require an in system/in situ/in operando X-ray spectroscopy approach to follow the growth of materials and the formation of functional interfaces as well as the evolution of their properties during successive device processing and operation. EMIL will employ multi-chamber small $\left(11 \times 11 \mathrm{~mm}^{2}\right)$ and large-area $(6$ " wafers) thin-film deposition tools across all relevant material classes, which are connected through ultra-high vacuum (UHV) transfer systems to advanced characterization tools to aid the development of real-world materials for PV, 
(photo)electrochemical, and (photo)catalytic devices. The analytical facilities feature a combination of complementary state-of-the-art X-ray analytical techniques in three end stations at a dedicated beamline delivering high-brilliance photons in the energy range of $80-10,000 \mathrm{eV}$. The availability of such a wide X-ray energy range from soft to hard X-rays will allow studying the physical and chemical properties of buried interfaces due to the easily tunable information depth accessible at the EMIL beamlines. Hard Xray energies further facilitate the study of liquid/solid interfaces in electrochemical processes like water splitting.

EMIL's technical specifications are pushing the limits of synchrotron-based X-ray analysis. The EMIL beamlines, to be commissioned in 2015, will provide a photon flux of up to $3 \times 10^{13}$ photons/s/0.1A/0.1\%BW and a resolution $\mathrm{E} / \Delta \mathrm{E}>$ 10,000. Spot sizes of $45 \times 10 \mu \mathrm{m}^{2}$ and $120 \times 10 \mu^{2}$ for soft and hard X-ray energies, respectively, will be realized (for details see [1]).

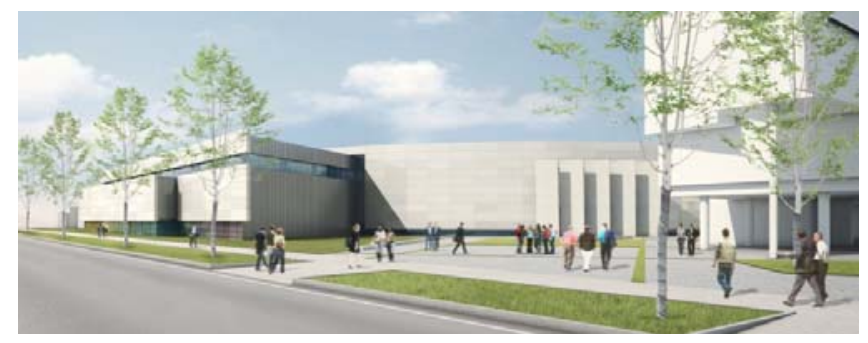

Fig. 1. The EMIL infrastructure at the BESSY II storage ring (courtesy of hammeskrause bda). For details and current status of the construction see http://www.helmholtz-berlin.de/projects/emil/.

The EMIL building is a separate infrastructure with a total floor area of $2000 \mathrm{~m}^{2}$ that is attached to the BESSY II synchrotron (see Fig. 1) with direct access to the dedicated Xray beamlines. EMIL will consist of two large laboratory infrastructures: SISSY (Solar Energy In situ Laboratory at the Synchrotron operated by HZB) and CAT (Catalysis Research for Sustainable Energy Supply operated by MPG). In particular, SISSY will feature a combination of the aforementioned state-of-the-art X-ray analytical techniques, and CAT will focus on fundamental research on catalytic processes under ambient conditions employing near-ambientpressure hard X-ray photoelectron spectroscopy. Additionally, in the CAT @EMIL laboratory near-ambient pressure HAXPES will be combined with in situ energy-dispersive XRD (ED-XRD). The simultaneous observation of surface and structural bulk properties during compound semiconductor film formation will thus be possible.

In addition, EMIL will have a chemistry lab and a clean room as well as a gas handling system that can supply the SISSY lab with up to 30 different gases (see Fig. 2). EMIL will be complemented by an electron microscopy facility with advanced focused electron and focused ion beam (Ga, Ne, He

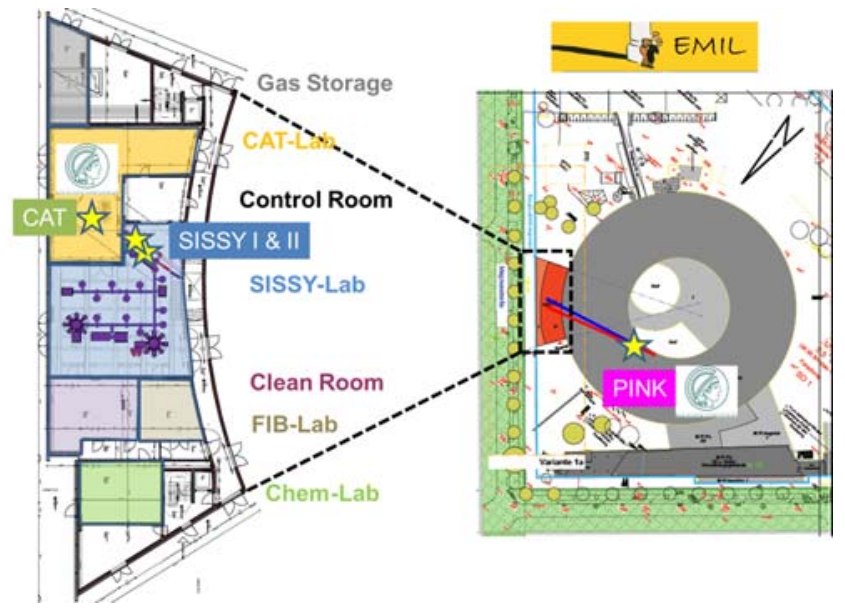

Fig. 2: Sketch of the laboratory layout of EMIL and its location with respect to the synchrotron.

ions) capabilities and analytical add-ons permitting correlated microscopies at defined locations, with nano-scale precision.

EMIL will have dedicated deposition and preparation facilities across all materials classes - thus explicitly allowing for hybrids and novel material combinations - as well as comprising combinatorial synthesis approaches so-far unrealized, Most of the aforementioned functionality will be hosted in a UHV system allowing for processing and analyzing up to 6” sized substrates which are handled using automated vacuum transfer systems, thus minimizing the time needed for sample transfer and thus reducing the probability of contamination, while enabling step-by-step deposition/analysis sequences. In its current planning phase EMIL will host a silicon deposition cluster tool with up to six deposition chambers that are directly connected to the automated UHV transfer line. The initial layout features two plasma-enhanced chemical vapor deposition (PECVD) chambers, one atomic layer deposition (ALD) chamber and one sputter deposition chamber, depositing on up to 6" size, i.e., spanning the range from "academic" samples up to fully industry-compatible wafers (see Fig. 3). These preparation facilities will be extended in the near future to include compound semiconductor processing for chalcopyrite, kesterite, and III-V materials, glove-box and vacuum-based organic semiconductor processing capabilities for organic PV (OPV), and hybrid concepts (perovskites). Furthermore, it is envisioned to add growth methods for emerging materials such as graphene, nanocomposites, and hybrid materials for solar spectrum shaping (photonic up- and down-conversion) or materials for (photo) catalysis. Also a characterization cluster with STM, AFM and micro-Raman setups will complement the analytic toolbox of EMIL.

By a pulsed laser deposition tool (PLD), EMIL will also enable composition-dependent libraries of the chemical and electronic structure of oxides with complex stoichiometry to 
be built for stable photoelectrodes for water splitting devices. By combining the lateral variation of stoichiometry realized by PLD in a large-area sample with spatially resolved X-ray analysis allows a much higher sample throughput. In addition, promising photoelectrodes will also be characterized in operando by near-ambient pressure HAXPES (in collaboration with MPG) in CAT @ EMIL. The stability and reactivity of such novel photoactive electrodes will be studied under real conditions close to the ambient pressure range. The aim is to detect in situ the surface/near-surface chemical composition during operation in the dark and under illumination.

The unique capabilities of EMIL's characterization/ preparation platform for in situ and in system analysis will enable both synergetic use of the unique synchrotron-based analysis environment and a cross-system approach in device design, explicitly envisioning hybrid organic/inorganic or combined PV/PEC devices.

\section{SUMMARY}

Up to now, a knowledge-based approach for optimizing solar energy devices is extremely time-consuming as the parameter space for material and device design is very large, and access to synchrotron-based analytics is limited. Due to the high reactivity of the involved materials and interfaces (e.g., for photovoltaic materials) and the very specific environment they are working in (e.g., in aqueous solution for catalysts), the mandatory in system, in situ, or in operando analysis of film growth or of devices under working conditions is in the core focus of the EMIL infrastructure. EMIL will therefore be a unique facility, enabling entirely new routes of energy materials development. EMIL will serve as a dedicated research platform and will be available for national and international collaboration.

EMIL is connecting synchrotron-based X-ray characterization techniques - such as X-ray photoelectron spectroscopy and -microscopy (XPS/XPEEM), as well as Xray diffraction (XRD), absorption (XAS) and emission/fluorescence (XES/XRF) spectroscopy with relevant in system/in situ sample preparation in one dedicated vacuum system.. EMIL will be dedicated to the in situ and in operando $\mathrm{X}$-ray analysis of materials and devices for photovoltaic applications and of (photo)catalytic/photo-electrochemical processes. EMIL is designed such that it can serve up to three experimental end-stations that each can simultaneously access soft and hard X-rays in an energy range of $80 \mathrm{eV}-10 \mathrm{keV}$,

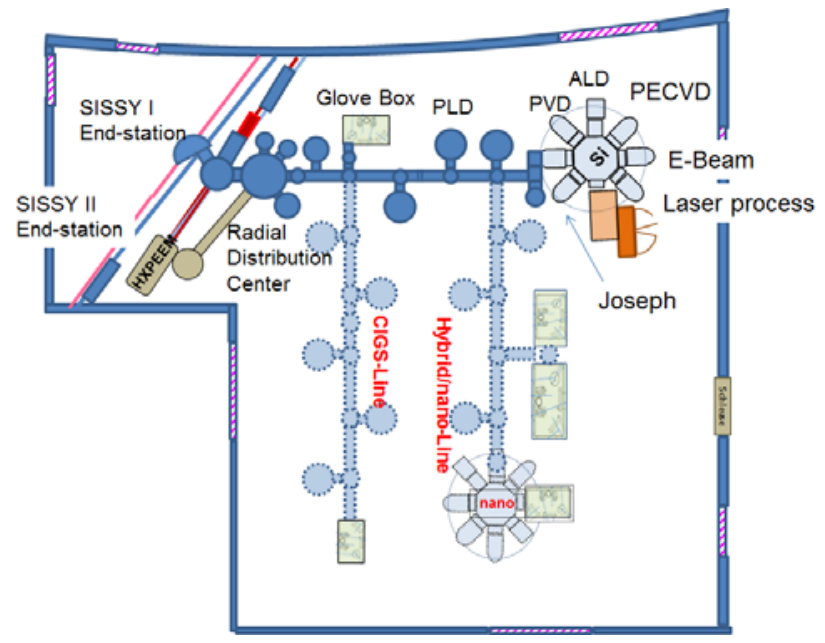

Fig 3.: Layout of the SISSY deposition and analytic laboratory. Blue and red lines at the left side represent the hard and soft X-ray

and comprises all characterization and deposition facilities in one integrated ultra-high vacuum system. In addition, a fourth end station is planned for dispersive XES using high flux radiation of 2-10 keV. Therefore, EMIL will serve as a research platform for national and international collaboration in the field of photovoltaic/photocatalytic energy conversion and beyond.

\section{ACKNOWLEDGEMENT}

We gratefully acknowledge funding through the Helmholtz Energy Materials Characterization Platform (HEMCP) initiated by the Helmholtz Association and the German Federal Ministry of Education and Research (BMBF). We are indebted to the Max-Planck Society for funding of the beamline and BMBF for providing resources through the project SISSY. M.B. also thanks the Impuls- und Vernetzungsfonds of the Helmholtz-Association (VH-NG423) for sponsorship.

\section{REFERENCES}

[1] R. Follath, M. Hävecker, G. Reichardt, K. Lips, J. Bahrdt, F. Schäfers, P. Schmidt, "The Energy Materials in situ Laboratory Berlin (EMIL) at BESSY II" in Proceedings of the 11th International Conference on Synchrotron Radiation Instrumentation (SRI 2012), Lyon, France. 